\title{
HPV RNA CISH score identifies two prognostic groups in a p16 positive oropharyngeal squamous cell carcinoma population
}

\author{
Jérémy Augustin (1) ${ }^{1}$ - Marion Mandavit ${ }^{2,3}$ - Sophie Outh-Gauer ${ }^{1}$ O Ophélie Grard ${ }^{2,3}$ - Cassandre Gasne ${ }^{2,4}$. \\ Charles Lépine $^{1} \cdot$ Haïtham Mirghani ${ }^{5} \cdot$ Stéphane Hans $^{4}$ - Pierre Bonfils ${ }^{4} \cdot$ Thomas Denize $^{1} \cdot$ Patrick Bruneval $^{1,2}$. \\ Justin A. Bishop ${ }^{6} \cdot$ Jacqueline Fontugne $^{7} \cdot$ Hélène Péréé $^{2,8} \cdot$ Eric Tartour $\mathbb{D}^{2,9} \cdot$ Cécile Badoual $^{1,2,3}$
}

Received: 28 February 2018 / Revised: 3 May 2018 / Accepted: 5 May 2018 / Published online: 20 June 2018

(c) United States \& Canadian Academy of Pathology 2018

\begin{abstract}
HPV-related and HPV-unrelated oropharyngeal squamous cell carcinomas are two distinct entities according to the Union for International Cancer Control, with a better prognosis conferred to HPV-related oropharyngeal squamous cell carcinomas. However, variable clinical outcomes are observed among patients with p16 positive oropharyngeal squamous cell carcinoma, which is a surrogate marker of HPV infection. We aimed to investigate the prognostic value of RNA CISH against E6 and E7 transcripts (HPV RNA CISH) to predict such variability. We retrospectively included 50 histologically confirmed p16 positive oropharyngeal squamous cell carcinomas (p16 positive immunostaining was defined by a strong staining in $70 \%$ or more of tumor cells). HPV RNA CISH staining was assessed semi-quantitatively to define two scores: RNA CISH "low" and RNA CISH "high". Negative HPV RNA CISH cases were scored as RNA CISH "low". This series contained 29 RNA CISH low cases (58\%) and 21 RNA CISH high cases (42\%). Clinical and pathologic baseline characteristics were similar between the two groups. RNA CISH high staining was associated with a better overall survival in both univariate and multivariate analyses ( $p=0.033$ and $p=0.042$, respectively). Other recorded parameters had no prognostic value. In conclusion, HPV RNA CISH might be an independent prognostic marker in p16 positive oropharyngeal squamous cell carcinomas and might help guide therapeutics.
\end{abstract}

Cécile Badoual

cecile.badoual@aphp.fr

1 Department of Pathology, Hôpital Européen Georges Pompidou, APHP, Paris, France

2 INSERM U970, Université Paris Descartes Sorbonne Paris-Cité, 75015 Paris, France

3 Equipe Labellisée Ligue Contre le Cancer, Paris, France

4 Department of ENT Surgery, Hôpital Européen Georges Pompidou, Paris, France

5 Department of ENT Surgery, Institut Gustave Roussy, Paris, France

6 Department of Pathology, UT Southwestern Medical Center, Dallas, TX, USA

7 Institut Curie, Research Center, 75005 Paris, France

8 Department of Microbiology, Hôpital Européen Georges Pompidou, Paris, France

9 Department of Immunology, Hôpital Européen Georges Pompidou, Paris, France
It is now established that high risk HPV infection drives carcinogenesis in a subset of oropharyngeal squamous cell carcinomas [1-3]. HPV-related oropharyngeal squamous cell carcinomas have a better prognosis than HPV-unrelated oropharyngeal squamous cell carcinomas and seem to be more sensitive to radiotherapy and radiochemotherapy [3-6]. This has motivated the development of organ sparing treatment strategies based on radiotherapy, currently evaluated through ongoing trials, with encouraging results [7-9]. As a consequence, the recent 8th Edition of the TNM Classification for Head and Neck Cancer (Union for International Cancer Control) separated p16 positive oropharyngeal squamous cell carcinomas and p16 negative oropharyngeal squamous cell carcinomas with two different stage classifications, acknowledging that HPV-related oropharyngeal squamous cell carcinomas constitute a distinct entity from HPV-unrelated oropharyngeal squamous cell carcinomas [10].

Several tools, including p16 immunostaining, are available to detect high risk HPV in head and neck squamous cell carcinomas. Among them, many authors showed that 
p16 immunostaining is a performant test in the oropharynx, with good sensitivity and negative predictive value. In fact, this cost-effective method is recommended as a surrogate marker of high risk HPV infection by the College of American Pathologists and by the Union for International Cancer Control [10, 11].

Although p16 immunostaining is a surrogate marker of high risk HPV infection, it may not reflect the heterogeneity of the HPV-induced oropharyngeal squamous cell carcinoma group, with variable clinical outcomes in p16 positive oropharyngeal squamous cell carcinoma populations. Indeed, some patients with p16-positive oropharyngeal squamous cell carcinoma experience locoregional treatment failure and may not respond to deintensification protocols [12-15]. Although p16 positive oropharyngeal squamous cell carcinomas have a better prognosis than p16 negative oropharyngeal squamous cell carcinomas, some clinicians observe variable clinical outcomes in p16 positive oropharyngeal squamous cell carcinoma populations. E6 and E7 proteins are encoded by high risk HPV and have transforming properties by respective disruption of the p53 and $\mathrm{pRB}$ signaling pathways [16]. Thus, detection of E6 and E7 transcripts is widely considered as the gold standard to identify an active HPV infection [17]. As the detection of E6 and E7 transcripts in oropharyngeal squamous cell carcinomas seems to be an independent predictor of superior overall survival in multivariate analysis [18-22], we hypothesized that different HPV E6 and E7 transcription levels could explain the variability of clinical courses in the subgroup of patients with a p16 positive oropharyngeal squamous cell carcinoma. Using a real time nucleic acid sequence based amplification, Jung et al. observed that HPV-positive oropharyngeal squamous cell carcinomas with E6/E7 transcripts had a significantly better overall survival than HPV-positive oropharyngeal squamous cell carcinomas with no E6/E7 transcripts [19]. Unfortunately, such assays cannot be performed easily in routine practice.

Chromogenic in situ hybridization against HPV RNA (HPV RNA CISH) offers excellent diagnostic performances to detect high risk HPV in oropharyngeal squamous cell carcinomas in routine practice on formalin-fixed paraffinembedded tissues [21, 23]. RNA molecule is known to be fragile. However, a recent study reported that RNA CISH can be used with satisfactory results on formalin-fixed paraffin-embedded tissues, with respect to positive control signals (Cyclophilin B gene expression), over time [24]. In this study, RNA CISH was performed prospectively and retrospectively on full sections from formalin-fixed paraffinembedded blocks (prospective collection) and on tissue micro arrays (retrospective collection). In the retrospective analysis, the number of Cyclophilin B signals per cell did not decrease over time (from 2004 to 2008). In only 3 out of 353 cases $(0.9 \%)$, no Cyclophilin B signal was identified, but this was not dependent on the age of the formalin-fixed paraffin-embedded block. Based on these observations, we decided to semi-quantitatively evaluate high risk HPV E6 and E7 expression levels using RNA CISH in a series of p16 positive oropharyngeal squamous cell carcinomas, with Cyclophilin B as a control probe.

The goal of this study was to determine the clinical relevance of HPV expression levels scored semiquantitatively using RNA CISH by evaluating its prognostic value in a series of 50 patients with p16 positive oropharyngeal squamous cell carcinoma.

\section{Materials and methods}

\section{Cases}

This monocentric retrospective study was approved by the Ethical Committee (Comité-de-Protection-des-Personnes Ile-de-France-II, ${ }^{\circ}$ 2015-09-04).

A total of 50 consecutive p16 positive oropharyngeal squamous cell carcinomas, diagnosed on biopsy or surgical specimen between 2011 and 2013, were retrieved from the Georges Pompidou European Hospital database. Inclusion criteria were: (i) histological confirmation of a newly diagnosed oropharyngeal squamous cell carcinoma, (ii) p16 positivity assessed by immunohistochemistry, and (iii) available tumor sample for HPV RNA CISH evaluation.

The following baseline characteristics were collected for each case: age at diagnosis, sex, $\mathrm{T}$ stage, $\mathrm{N}$ stage, smoking history (yes or no) and radiotherapy treatment (yes or no). Alcohol intake was not included in the analysis, because of insufficient collected data.

\section{Pathology and immunohistochemistry}

For each patient, a representative tumor slide from a formalin-fixed paraffin-embedded tissue block was selected to confirm tumor presence and to perform p16 immunostaining and HPV RNA CISH assay. All slides were reviewed by two pathologists (JA and CB).

Immunostaining against p16 protein was performed using a monoclonal p16 antibody (CINtec MTM; E6H4 clone) and p16 expression was revealed using the UltraView-Universal-Dab detection kit on a VentanaBenchMark Ultra autostainer (Ventana Medical Systems). Uterine cervix tissue was used as a positive control. A strong and diffuse cytoplasmic and nuclear staining in greater than $70 \%$ of malignant cells was reported as positive. An example of positive p16 immunostaining is shown in Fig. 1.

RNA CISH assay was performed to detect high risk HPV E6 and E7 transcripts (RNAscope 2.0 BROWN assay kit), 
Fig. 1 p16 positive oropharyngeal squamous cell carcinoma (a) showing a diffuse an intense immunostaining (b) (low magnification)
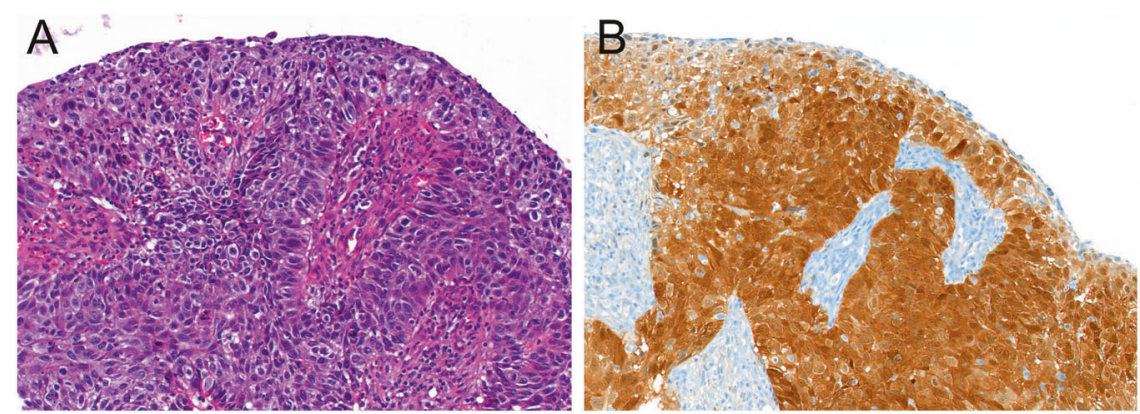

using the HPV-HR18 probe cocktail (Advanced Cell Diagnostics Inc.), according to the manufacturer's instructions. This cocktail contains probes targeting E6 and E7 transcripts from 18 high risk HPV genotypes (HPV16, $18,26,31,33,35,39,45,51,52,53,56,58,59,66,68$, 73 , and 82). A case was considered positive in the presence of a brown punctiform staining in the cytoplasm of tumor cells. The results were divided into two scores: RNA CISH "high" and RNA CISH "low". RNA CISH "high" score was defined by more than $50 \%$ of stained cancer cells, or by a staining covering more than $80 \%$ of the cell surface (nucleus and cytoplasm) in at least $30 \%$ of cancer cells at $\times 20$ objective. Lower staining and no staining were pooled in the "low" score group. Indeed, Dreyer et al. determined that RNA CISH was not reliable to discriminate between low amounts of E6/E7 transcripts and viral DNA in head and neck squamous cell carcinomas [25]. Based on this data, and because we hypothesized that HPV-related oropharyngeal squamous cell carcinomas with no or low levels of E6/E7 transcripts may have the same prognosis, we decided to aggregate cases with no or low RNA CISH expression into the same RNA CISH "low" group [25]. Examples of RNA CISH "high" and "low" scores are shown in Fig. 2. Positive control probes (Cyclophilin B housekeeping gene transcript) were used for each case and negative control probes (Bacillus subtilis dihydrodipicolinate reductase bacterial gene transcript) were used for 22 random cases. Staining with Cyclophilin B control probes was also assessed as "high" or "low" following the same rules as previously described, for positive cases. All stainings were blind-read independently by two pathologists (JA and $\mathrm{CB}$ ). For divergent cases (less than $10 \%$ ) a consensus reading on a multipipe microscope was organized.

\section{Polymerase chain reaction (PCR) for detecting HPV DNA}

DNA was extracted from tumoral formalin-fixed paraffinembedded shavings, using the QIAamp DNA minikit
(Quiagen), according to the manufacturer's instructions. Genotyping was performed using the INNO-Lipa Genotyping Extra Assay (Innogenetics). This assay is based on the principle of inverse hybridization and uses consensus SPF10 primers that amplify the L1 region of HPV DNA. This assay is able to detect 28 HPV genotypes (high risk: $16,18,26,31,33,35,39,45,51,52,53,56,58,59$, $66,68,73$, and 82 ; low risk: $6,11,40,43,44,54,70,69$, 71 , and 74).

\section{Follow-up}

Clinical endpoint for survival analysis was death. Survival time, in months, was defined by the delay from the time of diagnosis until the time of death.

\section{Statistical analysis}

Statistical analysis was performed using $\mathrm{R}$ software (R Foundation for Statistical Computing, 3.3.1-R Studio). Qualitative variables were compared using Fisher's exact test. Means were compared using Student $t$-test. The association between death and variables was evaluated using univariate and multivariate Cox proportional hazards regression models (R survival package). Variables with a $p$ value $<0.10$ and were selected as covariates for the multivariate analysis. Smoking history was also selected as a covariate because of its potential impact on survival. All tests were two-tailed and $p$ values $<0.05$ were considered statistically significant.

\section{Results}

\section{Population and tumors}

A total of 50 patients having a p16 positive oropharyngeal squamous cell carcinoma were included in this study. Among them, $76 \%$ were male. Mean age was 58 (range from 44 to 93 years). Tumor $\mathrm{T}$ stage and $\mathrm{N}$ stage were advanced (T3-T4 and $\mathrm{N}+$ ) in 63 and $96 \%$ of cases, 
Fig. 2 a, b RNA CISH "low" score stainings in oropharyngeal squamous cell carcinomas. a Low magnification and $\mathbf{b}$ high magnification: staining is observed in less than $50 \%$ of tumor cells and covers less than $80 \%$ of cell surface. $\mathbf{c}, \mathbf{d}$ RNA CISH "high" score stainings in oropharyngeal squamous cell carcinomas. c Low magnification and $\mathbf{d}$ high magnification: staining is observed in more than $50 \%$ of tumor cells and it this case, staining surface exceeds $80 \%$ in more than $30 \%$ of tumor cells
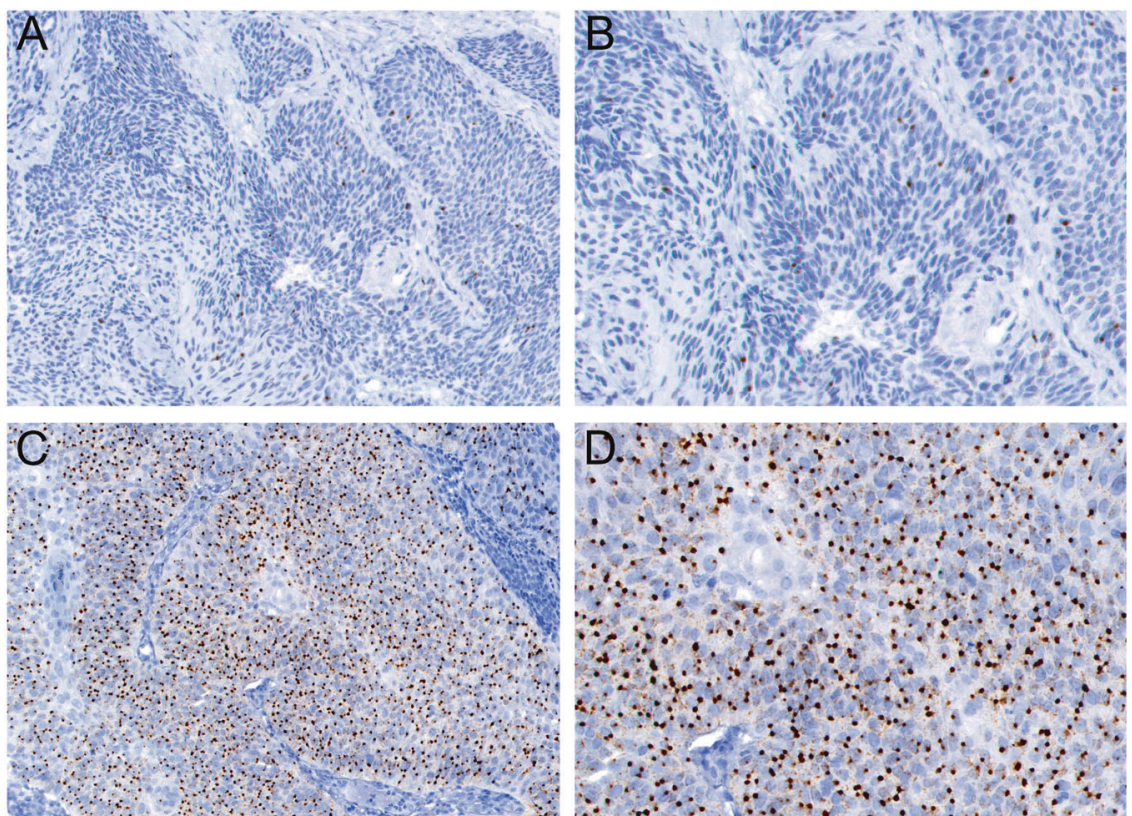

Table 1 Baseline clinicopathological characteristics of the study population, according to RNA CISH score

\begin{tabular}{|c|c|c|c|}
\hline & $\begin{array}{l}\text { RNA CISH } \\
\text { "low" }\end{array}$ & $\begin{array}{l}\text { RNA CISH } \\
\text { "high" }\end{array}$ & $p$ value \\
\hline Number of patients (\%) & $29(58)$ & $21(42)$ & \\
\hline Age (years) & & & 0.36 \\
\hline Mean & 58 & 58 & \\
\hline Range & $48-93$ & $44-73$ & \\
\hline $\operatorname{Sex}(n, \%)$ & & & 0.75 \\
\hline Female & $7(24)$ & $6(29)$ & \\
\hline Male & $22(76)$ & $15(71)$ & \\
\hline $\mathrm{T}$ stage $(n, \%)$ & & & 1 \\
\hline $\mathrm{T} 1-\mathrm{T} 2$ & $10(35)$ & $8(38)$ & \\
\hline $\mathrm{T} 3-\mathrm{T} 4$ & $18(62)$ & $13(62)$ & \\
\hline NA & $1(3)$ & & \\
\hline $\mathrm{N}$ stage $(n, \%)$ & & & 0.5 \\
\hline Negative & $2(7)$ & $0(0)$ & \\
\hline Positive & $26(90)$ & $21(100)$ & \\
\hline NA & $1(3)$ & & \\
\hline Smoking history $(n, \%)$ & & & 0.66 \\
\hline No & $7(24)$ & $8(38)$ & \\
\hline Yes & $22(76)$ & $13(62)$ & \\
\hline Radiotherapy $(n, \%)$ & & & 0.6359 \\
\hline No & $4(14)$ & $1(5)$ & \\
\hline Yes & $25(86)$ & $19(91)$ & \\
\hline
\end{tabular}

$N A$ data not available

respectively (8th TNM Union for International Cancer Control classification). A smoking history was present in $72 \%$ of cases. The clinical and pathological characteristics of the patients are summarized in Table 1 .
Table 2 HPV types detected in RNA CISH "high" and RNA CISH "low" groups

\begin{tabular}{ll} 
RNA CISH HPV “low” $(n=29)$ \\
\hline $\begin{array}{l}\text { Negative } \\
\text { staining }(n=11)\end{array}$ & Low positive \\
\end{tabular}

RNA CISH HPV "high" $(n$ $=21)$

\begin{tabular}{llll}
\hline HPV types (PCR) & & \\
HPV16 & $n=4(36 \%)$ & $n=15(83 \%)$ & $n=19(90 \%)$ \\
HPV26 & - & - & $n=1(5 \%)$ \\
HPV33 & - & $n=3(17 \%)$ & $n=1(5 \%)$ \\
No HPV & $n=7(64 \%)$ & - & - \\
detected & & & \\
\hline
\end{tabular}

\section{RNA CISH scores and HPV detection by PCR}

Among the 50 tumors, 29 (58\%) were RNA CISH low (including 11 cases with negative RNA CISH staining and 18 cases with low positivity), and 21 (42\%) were RNA CISH high. Control Cyclophilin B probes were visualized in all cases: 22 cases showed a low staining and 28 cases showed a high staining. To validate further survival analyses, we demonstrated that all cases with a negative RNA CISH HPV test displayed a positive Cyclophilin B control. Moreover, no statistical correlation between the grading of HPV RNA CISH and Cyclophilin B control stainings was identified (rho $=0,22$, Spearman rank correlation). A negative control probe (Bacillus subtilis dihydrodipicolinate reductase) was randomly performed on 22 of the 50 cases and all tested cases were negative. There were no differences between RNA CISH "low" and "high" groups regarding the baseline characteristics (Table 1). Particularly, 


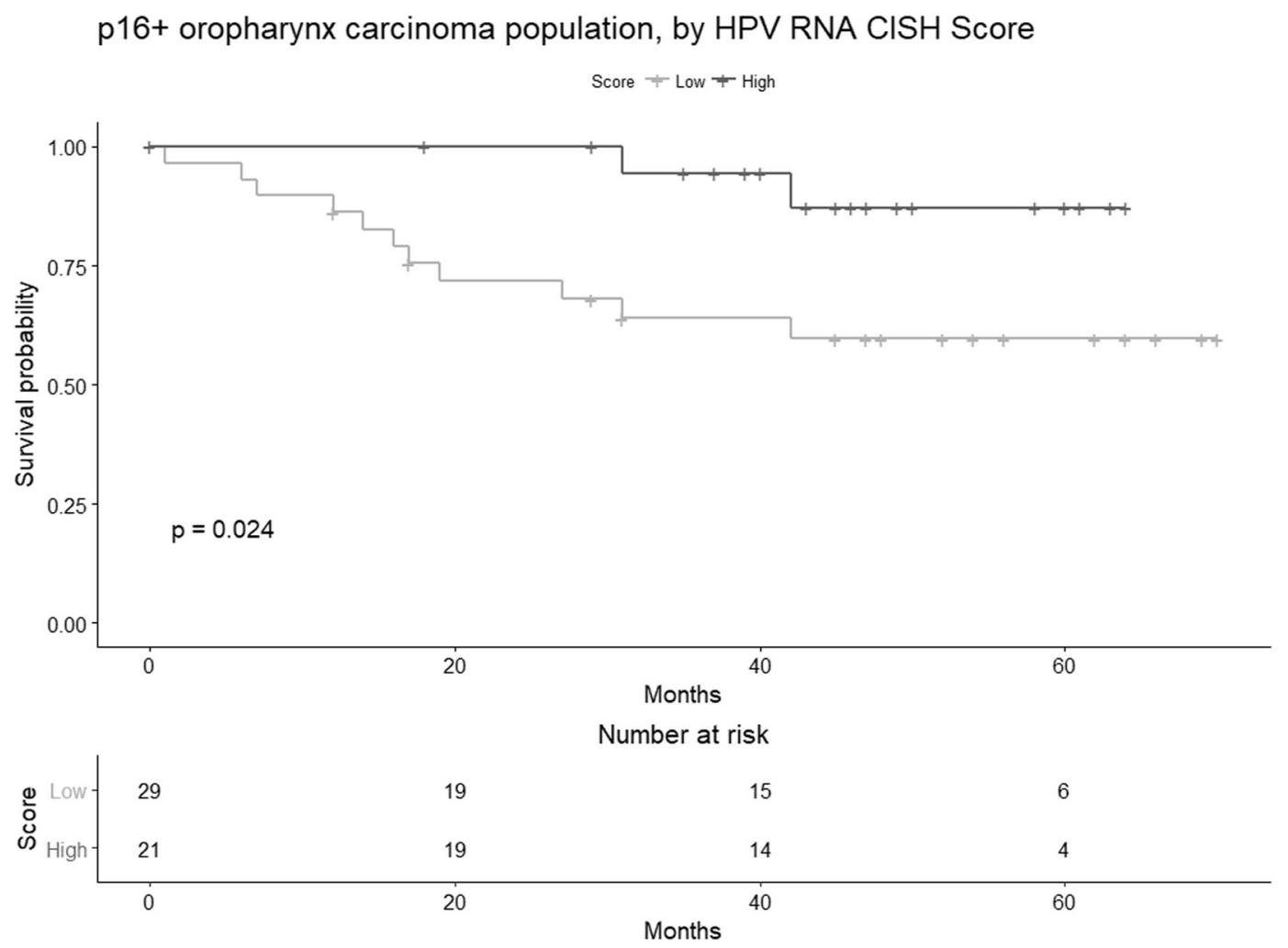

Fig. 3 Overall survival by RNA CISH score

patients with RNA CISH low tumors and patients with RNA CISH high tumors received radiotherapy in $86 \%$ and $91 \%$ of cases, respectively $(p=0.64)$. HPV types detected by PCR in RNA CISH high and RNA CISH low groups are reported in Table 2.

\section{p16 positive oropharyngeal squamous cell carcinomas with a RNA CISH high score have a better overall survival}

The median follow-up was 49 months (interquatile range 40-61). During follow-up, 13 deaths occurred, including 2 in the RNA CISH high group and 11 in the RNA CISH low group. In Kaplan-Meier analysis, patients with RNA CISH high tumors had a better overall survival than patients with RNA CISH low tumors ( $p=0.024$, log-rank test, Fig. 3). The 3-year overall survival rates were $64 \%$ (95\% IC, $48-85 \%$ ) in the RNA CISH low group and $94 \%$ (95\% IC, 84-100\%) in the RNA CISH high group (Fig. 3).

In univariate analysis (Cox model), the RNA CISH high group had a significantly better overall survival than the RNA CISH low group (Hazard ratio $=0.21, p=0.033$ ), and the other factors had no prognostic value, including smoking history $(p=0.23)$. However, there was a trend for a better survival for patients who had received radiotherapy $(p=0.07)$. In multivariate analysis, using radiotherapy and smoking history as covariates, the RNA CISH high group remained associated with a better overall survival $(p=$ 0.042 ), with a $76 \%$ reduction in the risk of death (adjusted hazard ratio $=0.24,95 \%$ IC, $6-95 \%$ ), whereas radiotherapy and smoking history were not statistically significant $(p=$ 0.21 and 0.47 , respectively). The results are reported in Table 3.

\section{Discussion}

As it is reported in the last edition of the TNM Classification for Head and Neck Cancer (Union for International Cancer Control), patients with p16 positive oropharyngeal squamous cell carcinomas have a better outcome than those with p16 negative oropharyngeal squamous cell carcinomas [10]. However, our study highlights that patients with a p16 positive oropharyngeal squamous cell carcinoma may represent a heterogenous population regarding survival. For the first time in literature, using an assay available in routine practice on formalin-fixed paraffin-embedded samples, we show that RNA CISH targeting E6 and E7 HPV transcripts might independently predict overall survival in p16 positive oropharyngeal squamous cell carcinomas.

Based on previous observations suggesting that negative and low expressions of RNA CISH should be gathered 
Table 3 Factors associated to risk of death in p16 positive oropharyngeal squamous cell carcinomas

\begin{tabular}{|c|c|c|c|c|}
\hline & Hazard ratio & $p$ value & $\begin{array}{l}\text { Adjusted hazard } \\
\text { ratio }\end{array}$ & $p$ value \\
\hline \multicolumn{5}{|c|}{ Explanatory variable } \\
\hline \multicolumn{5}{|l|}{ RNA CISH } \\
\hline Low & Ref & & Ref & \\
\hline High & $\begin{array}{l}0.21 \\
(0.05-0.88)\end{array}$ & 0.033 & $0.24(0.06-0.95)$ & 0.042 \\
\hline \multicolumn{5}{|l|}{ Sex } \\
\hline Female & Ref & & & \\
\hline Male & $1.24(0.33-4.6)$ & 0.752 & & \\
\hline \multicolumn{5}{|l|}{$\mathrm{T}$ stage $^{\mathrm{a}}$} \\
\hline $\mathrm{T} 1-\mathrm{T} 2$ & Ref & & & \\
\hline $\mathrm{T} 3-\mathrm{T} 4$ & $\begin{array}{l}1.687 \\
(0.45-6.28)\end{array}$ & 0.435 & & \\
\hline \multicolumn{5}{|l|}{$\mathrm{N}$ stage $^{\mathrm{a}}$} \\
\hline No & Ref & & & \\
\hline $\mathrm{N}+$ & Inf (0-Inf) & 1 & & \\
\hline \multicolumn{5}{|c|}{ Smoking history } \\
\hline No & Ref & & Ref & \\
\hline Yes & $2.6(0.5-11.9)$ & 0.231 & $\begin{array}{l}1.86 \\
(0.34-10.01)\end{array}$ & 0.47 \\
\hline \multicolumn{5}{|c|}{ Radiotherapy } \\
\hline No & Ref & & Ref & \\
\hline Yes & $\begin{array}{l}0.29 \\
(0.076-1.12)\end{array}$ & 0.07 & $0.41(0.07-2.31)$ & 0.31 \\
\hline \multicolumn{5}{|l|}{ Age (years) } \\
\hline \multicolumn{5}{|l|}{$<60$} \\
\hline$\geq 60$ & $\begin{array}{l}1.625 \\
(0.56-4.72)\end{array}$ & 0.371 & & \\
\hline
\end{tabular}

${ }^{\mathrm{a}}$ TNM classification (Union for International Cancer Control; 8th edition)

within the same group (RNA CISH low group) [25], we determined, in this series of p16 positive oropharyngeal squamous cell carcinomas with similar baseline characteristics, that the RNA CISH high group had a statistically significant better overall survival than RNA CISH low group, in both univariate and multivariate analyses. The clinical characteristics of our population of study was coherent with previous observations of p16 positive oropharyngeal squamous cell carcinomas, as we observed a mean age of 58 years, frequent tumor nodal involvement (96\%). Smoking habits tend to differ by populations but smoking history rate was relatively high in this French population $[2,26]$.

P16 immunostaining, with a diffuse and strong staining in more than $70 \%$ of cells, is a surrogate marker of active HPV infection and is recommended by the College of American Pathologists in oropharyngeal squamous cell carcinomas [11]. This threshold has been chosen because stainings in fewer than $70 \%$ of cells or with weak intensity can be observed in transcriptionally inactive HPV infection and in conditions not related to HPV infection (chronic inflammation, regeneration) [27]. However, the p16 positive oropharyngeal squamous cell carcinomas seem to be heterogenous on a biological level. Indeed, Wiest et al. showed that only a fraction of p16 positive and E6/E7 expressing oropharyngeal squamous cell carcinomas produce viralcellular fusion transcripts regarded as HPV integration, when occurring in the proximity of genes of potential interest is suggested to enhance carcinogenesis [16]. In the present study, we observed four p16 positive oropharyngeal squamous cell carcinomas with a negative RNA CISH HPV test but a positive PCR assay (all four cases harbored HPV type 16), highlighting the possible existence of high risk HPV infection with no associated transcription. Seven cases showed p16 positivity but RNA CISH and PCR negative tests, indicative of HPV unrelated p16 positivity (chronic inflammation, regeneration). According to Rietbergen et al., these HPV unrelated p16 positive cases would harbor p53 mutations more often than HPV-DNA positive cases [28]. In another work, Rietbergen et al. showed that patients with p16 positive but HPV-DNA negative oropharyngeal squamous cell carcinomas had a clinical course closer to HPV negative oropharyngeal squamous cell carcinomas than to HPV positive oropharyngeal squamous cell carcinomas [29]. The heterogeneity of p16 positive oropharyngeal squamous cell carcinomas has also been underlined by Holzinger et al. who reported that an active infection with high viral load conferred the lowest risk of death in a series of 199 oropharyngeal squamous cell carcinomas [30].

Several events may modulate E6 and E7 transcript rates in HPV positive tumors. As mentioned above, variable HPV viral loads may be observed in oropharyngeal squamous cell carcinomas and this could modify HPV-induced carcinogenesis as described in cervical cancer [30, 31]. Some epigenetic events could enhance E6 or E7 transcription, such as a hypomethylation of $\mathrm{p} 97$ promoter at the E6 proximal part of the non-coding locus control region present in the HPV genome [32]. Micro-RNAs (mir-9, miR181, and miR-375) were also shown to modulate E6 and E7 expression in HPV positive oropharyngeal squamous cell carcinomas. Additionally, HPV genome methylation has been described in HPV-related oropharyngeal squamous cell carcinomas [33-36] and histone modifications due to tobacco could modulate HPV carcinogenesis [37]. HPV integration into the host DNA enhances levels of E6 and E7 transcripts in cervical cancers [38]. Nevertheless, this process is not elucidated in head and neck squamous cell carcinomas and a recent study showed that HPV-induced oropharyngeal squamous cell carcinomas harbor integrated (24\%) or both integrated and episomal HPV (64\%), with no 
prognostic difference between these two groups [39]. Finally, as suggested by Ward et al. variable expressions of E6 and E7 proteins could modify antitumoral immune responses and participate in HPV-induced oropharyngeal squamous cell carcinoma heterogeneity. In their study, Ward et al. showed that, compared to low levels of tumorinfiltrating lymphocytes, high levels of tumor-infiltrating lymphocytes in oropharyngeal squamous cell carcinomas are associated with a better overall survival [40]. Altogether, these data suggest that E6 and E7 expression levels can vary and are not prone to be regarded as binary variables. P16 positive oropharyngeal squamous cell carcinoma seems to represent a heterogenous entity, comprised of cases with different E6 and E7 transcription levels.

The use of HPV RNA CISH in the diagnosis of an active HPV infection has been debated because of its high cost, compared to p16 immunostaining. On the one hand, the College of American Pathologists guidelines recommend the use of p16 as a surrogate marker of an active infection, and additional HPV-specific testing may be performed on the pathologist's own free will, in agreement with the treating clinician or in the context of a clinical trial [11]. On the other hand, some authors affirm that, even if more expensive, HPV RNA CISH is a reliable and reproducible assay, that can be used in routine practice as a standalone test to detect an active HPV infection [21, 23]. However, this technique is still emerging and not widely used, although time-saving [41]. Here, we supply data that suggests, for the first time, that HPV RNA CISH might provide additional information compared to p16 alone, to precise prognosis of patients with oropharyngeal squamous cell carcinoma. However, these results need to be confirmed on larger series. Moreover, computed assisted evaluation of E6 and E7 transcripts levels might help pathologists in the quantification of target spots.

In conclusion, we show that patients with p16 positive oropharyngeal squamous cell carcinoma with a RNA CISH HPV "high" score might have a better overall survival than patients with a RNA CISH HPV "low" score. These results, if confirmed, could better guide therapeutics and increase our comprehension of HPV biology in oropharyngeal squamous cell carcinoma carcinogenesis.

\section{Compliance with ethical standards}

Conflict of interest The authors declare that they have no conflict of interest.

\section{References}

1. D'Souza G, Kreimer AR, Viscidi R, et al. Case-control study of human papillomavirus and oropharyngeal cancer. N Engl J Med. 2007;356:1944-56.
2. Gillison ML, Koch WM, Capone RB, et al. Evidence for a causal association between human papillomavirus and a subset of head and neck cancers. J Natl Cancer Inst. 2000;92:709-20.

3. Taberna M, Mena M, Pavón MA, et al. Human papillomavirusrelated oropharyngeal cancer. Ann Oncol. 2017;28:2386-98.

4. Marur S, D'Souza G, Westra WH, et al. HPV-associated head and neck cancer: a virus-related cancer epidemic. Lancet Oncol. 2010;11:781-9.

5. Cheraghlou S, Yu PK, Otremba MD, et al. Treatment deintensification in human papillomavirus-positive oropharynx cancer: outcomes from the National. Cancer Data Base Cancer. 2018;124:717-26.

6. Lohaus F, Linge A, Tinhofer I, et al. HPV16 DNA status is a strong prognosticator of loco-regional control after postoperative radiochemotherapy of locally advanced oropharyngeal carcinoma: Results from a multicentre explorative study of the German Cancer Consortium Radiation Oncology Group (DKTK-ROG). Radiother Oncol. 2014;113:317-23.

7. Marur S, Li S, Cmelak AJ, et al. E1308: phase II trial of induction chemotherapy followed by reduced-dose radiation and weekly cetuximab in patients with HPV-associated resectable squamous cell carcinoma of the Oropharynx- ECOG-ACRIN Cancer Research Group. J Clin Oncol. 2017;35:490-7.

8. Lassen P, Lacas B, Pignon J-P, et al. Prognostic impact of HPVassociated p16-expression and smoking status on outcomes following radiotherapy for oropharyngeal cancer: the MARCH-HPV project. Radiother Oncol. 2018;126:107-15.

9. Kelly JR, Husain ZA, Burtness B. Treatment de-intensification strategies for head and neck cancer. Eur J Cancer. 2016;68:125-33.

10. Huang SH, O'Sullivan B. Overview of the 8th edition TNM classification for head and neck cancer. Curr Treat Options Oncol. 2017; $18: 40$.

11. Lewis JS, Beadle B, Bishop JA, et al. Human papillomavirus testing in head and neck carcinomas: guideline From the College of American Pathologists. Arch Pathol Lab Med. 2017;142:559-97. https://doi.org/10.5858/arpa.2017-0286-CP

12. O'Sullivan B, Huang SH, Siu LL, et al. Deintensification candidate subgroups in human papillomavirus-related oropharyngeal cancer according to minimal risk of distant metastasis. J Clin Oncol. 2013;31:543-50.

13. Maxwell JH, Kumar B, Feng FY, et al. Tobacco use in human papillomavirus-positive advanced oropharynx cancer patients related to increased risk of distant metastases and tumor recurrence. Clin Cancer Res. 2010;16:1226-35.

14. Kumar B, Cordell KG, Lee JS, et al. EGFR, p16, HPV Titer, Bcl-xL and $\mathrm{p} 53$, Sex, and smoking as indicators of response to therapy and survival in oropharyngeal cancer. J Clin Oncol. 2008;26:3128-37.

15. Vainshtein J, McHugh JB, Spector ME, et al. Human papillomavirus-related oropharyngeal cancer: HPV and p16 status in the recurrent versus parent tumor: HPV-related cancer in parent versus recurrent tumors. Head Neck. 2015;37:8-11.

16. Wiest T, Schwarz E, Enders C, et al. Involvement of intact HPV16 E6/E7 gene expression in head and neck cancers with unaltered p53 status and perturbed pRb cell cycle control. Oncogene. 2002;21:1510-7.

17. Ndiaye C, Mena M, Alemany L, et al. HPV DNA, E6/E7 mRNA, and p16INK4a detection in head and neck cancers: a systematic review and meta-analysis. Lancet Oncol. 2014;15:1319-31.

18. Shi W, Kato H, Perez-Ordonez B, et al. Comparative prognostic value of HPV16 E6 mRNA compared with in situ hybridization for human oropharyngeal squamous carcinoma. J Clin Oncol. 2009;27:6213-21.

19. Jung AC, Briolat J, Millon R, et al. Biological and clinical relevance of transcriptionally active human papillomavirus (HPV) infection in oropharynx squamous cell carcinoma. Int $\mathbf{J}$ Cancer. 2010;126:1882-94. 
20. Kuo K-T, Hsiao C-H, Lin C-H, et al. The biomarkers of human papillomavirus infection in tonsillar squamous cell carcinomamolecular basis and predicting favorable outcome. Mod Pathol. 2008;21:376-86.

21. Bishop JA, Ma X-J, Wang H, et al. Detection of transcriptionally active high-risk HPV in patients with head and neck squamous cell carcinoma as visualized by a novel E6/E7 mRNA in situ hybridization method. Am J Surg Pathol. 2012;36:1874-82.

22. Mirghani H, Casiraghi O, Guerlain J, et al. Diagnosis of HPV driven oropharyngeal cancers: comparing p16 based algorithms with the RNAscope HPV-test. Oral Oncol. 2016;62:101-8.

23. Mirghani H, Casiraghi O, Amen F, et al. Diagnosis of HPV-driven head and neck cancer with a single test in routine clinical practice. Mod Pathol. 2015;28:1518-27.

24. Bingham V, McIlreavey L, Greene C, et al. RNAscopein situhybridization confirms mRNA integrity in formalin-fixed, paraffinembedded cancer tissue samples. Oncotarget. 2017;8:93392-403.

25. Dreyer JH, Hauck F, Oliveira-Silva M, et al. Detection of HPV infection in head and neck squamous cell carcinoma: a practical proposal. Virchows Arch. 2013;462:381-9.

26. Ang KK, Harris J, Wheeler R, et al. Human papillomavirus and survival of patients with oropharyngeal cancer. N Engl J Med. 2010;363:24-35.

27. Khleif SN, DeGregori J, Yee CL, et al. Inhibition of cyclin DCDK4/CDK6 activity is associated with an E2F-mediated induction of cyclin kinase inhibitor activity. Proc Natl Acad Sci USA. 1996;93:4350-4.

28. Rietbergen MM, Snijders PJF, Beekzada D, et al. Molecular characterization of p16-immunopositive but HPV DNA-negative oropharyngeal carcinomas: characterisation of p16immunopositive OPSSCs. Int J Cancer. 2014;134:2366-72.

29. Rietbergen MM, Brakenhoff RH, Bloemena E, et al. Human papillomavirus detection and comorbidity: critical issues in selection of patients with oropharyngeal cancer for treatment deescalation trials. Ann Oncol. 2013;24:2740-5.

30. Holzinger D, Schmitt M, Dyckhoff G, et al. Viral RNA patterns and high viral load reliably define oropharynx carcinomas with active HPV16 involvement. Cancer Res. 2012;72:4993-5003.
31. Dong L, Wang MZ, Zhao X, et al. Human papillomavirus viral load as a useful triage tool for non-16/18 high-risk human papillomavirus positive women: a prospective screening cohort study. Gynecol Oncol. 2018;148:103-10.

32. Park I-S, Chang X, Loyo M, et al. Characterization of the methylation patterns in human papillomavirus type 16 viral DNA in head and neck cancers. Cancer Prev Res Phila. 2011;4:207-17.

33. Wald AI, Hoskins EE, Wells SI, et al. Alteration of microRNA profiles in squamous cell carcinoma of the head and neck cell lines by human papillomavirus. Head Neck. 2011;33:504-12.

34. Jung H, Phillips BL, Chan EK. miR-375 activates p21 and suppresses telomerase activity by coordinately regulating HPV E6/ E7, E6AP, CIP2A, and 14-3-3ఢ. Mol Cancer. 2014;13:80.

35. Lajer CB, Nielsen FC, Friis-Hansen L, et al. Different miRNA signatures of oral and pharyngeal squamous cell carcinomas: a prospective translational study. $\mathrm{Br} \mathrm{J}$ Cancer. 2011;104:830-40.

36. Boscolo-Rizzo P, Furlan C, Lupato V, et al. Novel insights into epigenetic drivers of oropharyngeal squamous cell carcinoma: role of HPV and lifestyle factors. Clin Epigenetics. 2017;9:124.

37. Papillon-Cavanagh S, Lu C, Gayden T, et al. Impaired H3K36 methylation defines a subset of head and neck squamous cell carcinomas. Nat Genet. 2017;49:180-5.

38. Cullen AP, Reid R, Campion M, et al. Analysis of the physical state of different human papillomavirus DNAs in intraepithelial and invasive cervical neoplasm. J Virol. 1991;65:606-12.

39. Lim MY, Dahlstrom KR, Sturgis EM, et al. Human papillomavirus integration pattern and demographic, clinical, and survival characteristics of patients with oropharyngeal squamous cell carcinoma. Head Neck. 2016;38:1139-44.

40. Ward MJ, Thirdborough SM, Mellows T, et al. Tumourinfiltrating lymphocytes predict for outcome in HPV-positive oropharyngeal cancer. Br J Cancer. 2014;110:489-500.

41. National Collaborating Centre for Cancer (UK). Cancer of the upper aerodigestive tract: assessment and management in people aged 16 and over. London: National Institute for Health and Care Excellence (UK); 2016. http://www.ncbi.nlm.nih.gov/books/ NBK344100/. Accessed 28 February 2018. 